(b) the nicotine-containing liquid does not contain nicotine in excess of $20 \mathrm{mg} / \mathrm{ml}$ '." This error has also been addressed as an Author Correction in this issue of the European Respiratory Journal.

@ERSpublications

A correction on electronic nicotine delivery systems http://ow.ly/EWkzj

Francesco Blasi ${ }^{1}$ and Brian Ward ${ }^{2}$

${ }^{1}$ Dept of Pathophysiology and Transplantation, University of Milan, IRCCS Fondazione Cà Granda Milano, Milan, Italy.

${ }^{2}$ European Affairs Dept, European Respiratory Society, Brussels, Belgium.

Correspondence: Francesco Blasi, Dept of Pathophysiology and Transplantation, University of Milan, IRCCS Fondazione Cà Granda Milano, Via Francesco Sforza 35, 20122 Milan, Italy. E-mail: francesco.blasi@unimi.it

Received: Nov 212014 | Accepted: Nov 212014

Conflict of interest: Brian Ward is an employee of the European Respiratory Society.

References

1 Blasi F, Ward B. Electronic nicotine delivery systems (ENDS): the beginning of the end or the end of the beginning? Eur Respir J 2014; 44: 585-588.

2 Directive 2014/40/EU of the European Parliament and of the Council of 3 April 2014 on the approximation of the laws, regulations and administrative provisions of the Member States concerning the manufacture, presentation and sale of tobacco and related products and repealing Directive 2001/37/EC. Off J Eur Union 2014; L127: 1-38.

\title{
Glucocorticoids induce the production of the chemoattractant CCL20 in airway epithelium
}

To the Editor:

We read with interest the report by ZiJLSTRA et al. [1] in which the effects of glucocorticoids possibly contributed to airway neutrophilia in asthma. This study nicely adds to the research demonstrating that corticosteroids not only inhibit the production of several inflammatory chemokines and cytokines, but also corticosteroids increase levels of certain regulatory proteins; these neutrophil-active proteins are potentially involved in inflammation or, indeed, in host defence. Hence, the data reported by ZiJLSTRA et al. [1] may deserve attention beyond the focus given by the authors.

Neutrophils are present in sputum of normal subjects. They are also increased in many respiratory conditions. Unsurprisingly, striking variability of sputum neutrophil counts has been demonstrated in corticosteroid-treated asthma [2] and was also recorded by ZijLSTRA et al. [1]. The notion that corticosteroids cause neutrophilia by inhibiting apoptosis of these cells is flourishing in the literature. It is so established that there is no longer any need to present supporting evidence [3]. ZIJLSTRA et al. [1] do not discuss this aspect but their choice of reference regarding neutrophilic asthma is focussed on popular roles of apoptosis.

In vitro data have, for the last two decades, suggested the possibility that corticosteroids may reduce neutrophil apoptosis. Yet, there are no known data that compellingly support a role of this pharmacological treatment in patients; quite the opposite: in a careful biopsy study, GIZYCKI et al. [4] could not find any effect of corticosteroid treatment on neutrophil apoptosis compared with placebo treatment in chronic obstructive pulmonary disease. Furthermore, UDDIN et al. [5] excluded a role of corticosteroid treatment as a factor in pro-survival activity for airway neutrophils in severe asthma. Lack of support for apoptosis-related effects actually lends weight to the findings of ZiJLSTRA et al. [1], suggesting that a chemoattractant such as CCL20 could be involved in corticosteroid-induced airway neutrophilia.

On this note, it is of interest that severe asthma is associated with upregulation of CXCL5, possibly caused in part by corticosteroid treatment [6]. Furthermore, FuKaKusA et al. [7] demonstrated that systemic 
corticosteroids significantly upregulated interleukin-8, interferon- $\gamma$-inducible protein 10 and monocyte chemotactic protein-2 in asthmatic bronchial walls. Hence, in addition to CCL20, reported and discussed by ZIJLSTRA et al. [1], corticosteroids may upregulate several neutrophil-active chemokines in asthmatic bronchi. Work from different laboratories demonstrates reduced lumen neutrophils along with bronchial wall neutrophilia in corticosteroid-treated patients (reviewed in [8]). In these cases, corticosteroid-induced neutrophil attractants $[5,6]$ may have retained neutrophils in the bronchial wall, preventing their elimination by the transepithelial exit route [8].

We suggest that increased chemoattractants are more likely than apoptosis inhibition to contribute to bronchial neutrophilia in asthmatics receiving corticosteroids. What then is the role of corticosteroid-induced bronchial neutrophils?

The notion that neutrophils are pathogenic in severe asthma is intriguing but awaits validation by selective treatments. However, a role of neutrophils in host defence is undisputed. We and others have noted that the anti-inflammatory pharmacology of glucocorticoids may spare several inflammation-like, innate immunity events including plasma exudation and neutrophilia associated with airway infection and repair [8]. Supporting the possibility of a role of neutrophils in asthmatic airway defence, sputum numbers of these cells appear disconnected to other measures of airway inflammation in steroid-treated asthma $[2,8]$. Perhaps the glucocorticoid-induced expression of neutrophil attractants $[1,6,7]$ is a functional innate immunity-enhancing effect. This aspect would add to the mechanisms reported by ZHANG et al. [9], who have advanced the view that corticosteroids spare or enhance several mechanisms of protective innate immunity.

Novel corticosteroid-related [10] and -unrelated [1] molecular mechanisms are frequently and importantly suggested as future anti-asthma/anti-inflammatory drug targets. However, a major challenge is to distinguish between good and bad aspects of inflammation. Hence, anti-asthma drug discovery research would probably be helped by improved understanding of the balance between inhibition of disease-driving inflammation and stimulation of protective innate immunity by corticosteroids.

@ERSpublications

A major challenge is to distinguish between good and bad aspects of inflammation http://ow.ly/DAglc

Carl Persson ${ }^{1}$ and Lena Uller ${ }^{2}$

${ }^{1}$ Dept of Clinical Pharmacology, Laboratory of Medicine, Lund University Hospital, Lund, Sweden. ${ }^{2}$ Dept of Experimental Medical Science, Unit of Respiratory Immunopharmacology, Lund University, Lund, Sweden.

Correspondence: Carl Persson, Dept of Clinical Pharmacology, Laboratory of Medicine, Lund University Hospital, S-22185 Lund, Sweden. E-mail: Carl.persson@med.lu.se

Received: Sept 292014 | Accepted: Oct 162014

Conflict of interest: None declared.

\section{References}

1 Zijlstra GJ, Fattahi F, Rozeveld D Glucocorticoids induce the production of the chemoattractant CCL20 in airway epithelium. Eur Respir J 2014; 44: 361-370.

2 Arron JR, Choy DF, Laviolette M Disconnect between sputum neutrophils and other measures of airway inflammation in asthma. Eur Respir J 2014; 43: 627-629.

3 Persson C. Airway, apoptosis, and asthma. Clin Exp Allergy 2013; 43: 1083-1085.

4 Gizycki MJ, Hattotuwa KL, Barnes N Effects of fluticasone propionate on inflammatory cells in COPD: an ultrastructural examination of endobronchial biopsy tissue. Thorax 2002; 57: 799-803.

5 Uddin M, Nong G, Ward J Prosurvival activity for airway neutrophils in severe asthma. Thorax 2010; 65: 684-689.

6 Qiu Y, Zhu J, Bandi V Bronchial mucosal inflammation and upregulation of CXC chemoattractants and receptors in severe exacerbations of asthma. Thorax 2007; 62: 475-482.

7 Fukakusa M, Bergeron C, Tulic MK Oral corticosteroids decrease eosinophil and CC chemokine expression but increase neutrophil, IL-8, and IFN- $\gamma$-inducible protein 10 expression in asthmatic airway mucosa. J Allergy Clin Immunol 2005; 115: 280-286.

8 Persson C, Uller L. Transepithelial exit of leucocytes: inflicting, reflecting or resolving airway inflammation? Thorax 2010; 65: 1111-1115.

9 Zhang N, Truong-Tran QA, Tancowny B Glucocorticoids enhance or spare innate immunity: effects in airway epithelium are mediated by CCAAT/enhancer binding proteins. J Immunol 2007; 179: 578-589.

10 Barnes PJ. Corticosteroid resistance in patients with asthma and chronic obstructive pulmonary disease. J Allergy Clin Immunol 2013; 131: 636-645. 